\title{
Maintaining Euthyroidism: Fundamentals of Thyroid Hormone Physiology, lodine Metabolism and Hypothyroidism
}

\author{
De Wet Wolmarans* \\ Division of Pharmacology, Center of Excellence for Pharmaceutical Sciences, Faculty of Health Sciences, North West-University, \\ Potchefstroom, South Africa
}

*Corresponding author, email:dewet.wolmarans@nwu.ac.za

\begin{abstract}
Thyroid-related pathologies, especially subclinical and clinical hypothyroidism, are commonly described in clinical practice. While illnesses related to aberrant thyroid hormone homeostasis are the most prevalent endocrinological conditions diagnosed, important aspects related to thyroid hormone physiology are often overlooked. Further, the only known physiological purpose of iodine is its requirement for the synthesis of thyroxine (T4) and triiodothyronine (T3). However, although it can be applied as curative agent in the management of thyrotoxicosis, the halogen is often prescribed and used inappropriately with significant, but preventable clinical consequences. In an attempt to kindle a better understanding of aspects related to aberrant thyroid hormone regulation, iodine metabolism, and the clinical management of thyroid related pathology, the current paper provides a physiological and clinical overview of said constructs from a hypothyroid perspective.
\end{abstract}

\section{Lost Puzzle Pieces: A Brief Introduction to Thyroid Hormone Physiology}

What begins as a rapidly changing process of concentrating iodide already by the 11 th week of gestation, quickly matures to become one of the most important endocrine systems in the human body. In fact, while thyroid hormones, i.e. triiodothyronine (T3) and thyroxine (T4), play a critical role in facilitating normal growth and development, they also drive and regulate a vast number of physiological processes throughout adulthood. ${ }^{1}$ These include, among others, central nervous system (CNS) development, central and peripheral nervous system signaling, cardiac function, nutrient metabolism, hepatic metabolism, energy consumption and regulation of body temperature, water and electrolyte balance, and skeletal turnover. ${ }^{1}$ Consisting of two lobes, each approximately $2.0 \times 2.5 \times 4.0 \mathrm{~cm}$, the thyroid weighs on average $15-20 \mathrm{~g}$. Functionally, the gland consists of spherical follicles, each composed of a single layer of epithelium surrounding a colloid-containing lumen. Whereas the epithelium is responsible for the synthesis of $\mathrm{T} 3$ and T4, the colloid mainly consists of thyroglobulin (TG), a glycoprotein capable of storing thyroid hormone and its precursors, monoiodotyrosine (MIT) and diiodotyrosine (DIT).

\subsection{A concise overview of T3 and T4 synthesis and the roles of thyrotropin (TSH) and iodine}

Although an in-depth overview of thyroid hormone physiology falls outside the scope of the current review, certain key aspects of relevance for clinical practice will be discussed here.
The production and release of $\mathrm{T} 3$ and $\mathrm{T} 4$ involves four processes, i.e. uptake and oxidation of iodine, iodination of tyrosine, synthesis of $\mathrm{T} 3$ and $\mathrm{T} 4$, and ultimately resorption and proteolysis of TG. All four processes are intrinsically related to the availability of iodine and thyroid stimulating hormone (thyrotropin, TSH). To produce T4 at the normal rate of $85 \mu \mathrm{g}$ per day, a thyroidal uptake of $75 \mu \mathrm{g}$ iodine is necessary. To compensate for loss via renal and fecal excretion, the recommended daily intake values for iodine are 100-150 $\mathrm{gg}$ in adults, 90-120 $\mu \mathrm{g}$ in children and $200 \mu \mathrm{g}$ in pregnant women. ${ }^{1}$ However, across most of the world, including South Africa, sufficient quantities of iodine are provided by diet and water intake, negating the need for supplementation. Importantly, whereas the total content of iodine in the peripheral pool amounts to approximately $250 \mu \mathrm{g}$, the thyroid gland contains up to $8000 \mu \mathrm{g}$, most of which is in the form of MIT and DIT. Under a state of euthyroidism, each TG will contain at least 3-4 T4 molecules, while only 1 in 5 TGs will contain a single T3, accounting for an intrathyroidal T3:T4 ratio of 1:15. However, in the absence of sufficient thyroidal iodine, the T3:T4 ratio increases, probably due to $25 \%$ less iodine being necessary to synthesize T3, compared to T4. ${ }^{5}$ Still, considering the vast intrathyroidal iodine stores, such change in the T3:T4 ratio will only become apparent after approximately $40-50$ days of iodine deficiency. ${ }^{6}$

Normally, when the plasma free fraction of T4 (fT4) decreases, the hypothalamus and pituitary respond by secreting thyrotropin releasing hormone (TRH) and TSH, respectively. TSH subsequently acts by increasing the thyroid uptake of iodide via the sodium-iodide symporter (NIS), while potentiating the intracellular oxidation processes needed for hormone 


\begin{abstract}
Info Frame 1: Too much of a good thing: The matter of iodine
The only known function of iodine in human physiology concerns its involvement in normal thyroid hormone synthesis. For this purpose, a minimum dietary intake of $90-200 \mu \mathrm{g}$ of elemental iodine is necessary. Moreover, as explained in paragraphs 1.1-1.3, the body is able to respond to iodine deficiency by 1) increasing the synthesis ratio of T3:T4, 2) increasing TSH release and bolstering the actions of thyroid peroxidase (TPO) and the sodium-iodide symporter (NIS), 3) upregulating the expression of iodothyronine deiodinase type $2\left(D_{2}\right)$, while downregulating the activity of $D_{1}$ and $D_{3}$, increasing the extent of dissociation between thyroid binding globulin (TBG) and T3/T4, and 5) reducing the clearance rate of both T3 and T4.

However, while such mechanisms can be deployed to sustain a relatively euthyroid state in conditions of iodine deficiency, the same cannot be stated in case of iodine excess. First, although the thyroid can compensate for the effects of acute increases in iodine by preventing the release of stored T3 and T42 and interrupting the iodination of tyrosine at high intracellular concentrations (Wolff-Chaikoff effect), ${ }^{3}$ these responses cannot be sustained. Eventually, following continued administration of supraphysiological doses of iodine, the anion prevents its own uptake, possibly by inhibiting the actions of the NIS. ${ }^{4}$ Consequently, escape from the Wolff-Chaikoff effect is induced with rebound hyperthyroidism being a possibility. Importantly, the iodine dose needed to induce the Wolff-Chaikoff effect can range from 18-54 mg per day, while any amount between this and the recommended daily intake can induce hyperthyroidism from the outset.

Apart from its effects on thyroid physiology, chronic excessive iodine exposure elicits a biological response similar to epithelial inflammation. In what is often described as 'iodism,' patients may present with coryza, rhinitis, skin rash, sialadenitis and excessive salivation, conjunctivitis, vasculitis, and eosinophilic granulocytosis.'

In South Africa, iodine is readily available over the counter, either in the form of saturated solutions e.g. Lugol's solution, as antibacterial preparations, e.g. povidone iodine, or as a constituent of multi-ingredient formulas, e.g. kelp tablets, multivitamin preparations, or iodized throat lozenges. With the exception of the latter, most of the multi-ingredient preparations contain iodine in amounts that agree with the recommended daily intake values. On the other hand, one drop of Lugol's solution provides $6000 \mathrm{\mu g}$ elemental iodine, a 30-fold increase over that of the recommended amount. Thus, if such a concentration of iodine is excessive and potentially harmful, the question is asked why Lugol's solution is available? The answer is founded on the basis of its thyroidal actions. As alluded to above, iodine in doses of 18-54 mg divided over 24 hours, inhibits thyroidal T3/T4 release and prevents the iodination of tyrosine. As iodine is the only known compound that can elicit such a rapid response, Lugol's solution is intended for use in thyrotoxic emergencies. However, to prevent escape-related rebound hyperthyroidism, iodine therapy is to be initiated with hormone synthesis inhibitors, e.g. carbimazole, and must be withdrawn after a maximum treatment duration of 10-14 days.

Taken from the above and considering that South Africans in general do not suffer from iodine deficiency, that iodine has no other physiological function apart from maintaining normal thyroid physiology and that no empirical evidence exists that supports the use of systemic iodine for infective states, the systemic use of Lugol's solution either as an iodine supplement or to support thyroid function in states of clinical or sub-clinical hypothyroidism, is strongly discouraged. In fact, the prescription and use of Lugol's solution for these purposes, is inappropriate. Where patients are suspected to present with clinical or sub-clinical hypothyroidism caused by iodine deficiency, a change in diet or iodine supplementation can be advised. It can be argued that one drop of Lugol's solution dissolved in a glass of water of which the patient can administer 5-10 m/ per day, provides iodine in concentrations analogous to the recommended daily intake. However, given the strength of the solution, that a number of other cost-effective preparations are available and that patients are often not sensitive to or informed of the biological chemistry of iodine, such arguments are irresponsible.
\end{abstract}

synthesis. ${ }^{7}$ The latter are all dependent on sufficient levels of thyroid peroxidase (TPO), also generated in response to TSH stimulation. Considering the functional crosstalk between the TSH receptor (TSHR), TPO and TG in the synthesis of thyroid hormone, it is noteworthy that all three components are targeted by autoimmune mechanisms in patients susceptible to chronic thyroiditis. ${ }^{8}$ Generally, in the absence of hypothalamic or pituitary dysfunction, the relationship between fT4 and TSH is inversely correlated to such an extent that measurements of plasma TSH almost always provide an accurate, albeit indirect indication of thyroid functioning. Whereas a pulsatile secretion of TSH with a wave frequency of 1-2 hours is normally sufficient for adequate T4 release, a number of factors can modify this response. For example, the pulse amplitude is significantly attenuated during illness, fasting or after surgery. ${ }^{9,10}$ If laboratory assessments of thyroid function during such conditions are interpreted, this must be taken into consideration. Apart from the significant inverse correlation between TSH and fT4 - and that fT4 is the major determinant of TSH secretion - TSH release is also modified by acute administration of somatostatin and dopaminergic agents. Indeed, both somatostatin and its analogues, e.g. octreotide (Sandostatin ${ }^{\circ}$ ), as well as dopaminergic potentiators, e.g. cabergoline (Dostinex $\left.{ }^{\circ}\right)$, I-dopa (Carbilev ${ }^{\circ}$ ), and quinagolide (Norprolac') inhibit the release of TSH. The opposite is also true in that administration of centrally acting dopamine antagonists, e.g. metoclopramide (Clopamon ${ }^{\circ}$ ), risperidone $\left(\right.$ Risperdal $\left.{ }^{\circ}\right)$, and chlorpromazine (Largactil $\left.{ }^{\circ}\right)$, may bolster TSH secretion. Interestingly, no lasting changes in TSH release result from either interference, indicating that at least in patients with a functioning hypothalamus-pituitary axis, compensatory mechanisms prevent TSH-related pathophysiological responses to said treatments.

Info Frame 2: Keynotes on $\mathrm{TSH}^{1}$
- Facilitates all steps of thyroid hormone synthesis
- Biological t¹/2 = 30 min
- Normal serum concentration: $0.4-4.2 \mathrm{mU} / \mathrm{L}$; inversely related
to thyroid function; concentration will increase in primary
hypothyroidism and decrease during primary hyperthyroidism
- TSHR susceptible to binding by stimulating, antagonizing and neutral
antibodies
- TSH shares homology with luteinizing hormone $(\mathrm{LH})$ and human
chorionic gonadotropin (hCG) and cross stimulation may occur,
especially during pregnancy

\subsection{The concepts of plasma transport and free fraction}

Given their lipophilic nature, thyroid hormones are extensively bound to plasma proteins, i.e. thyroxine binding globulin (TBG), transthyretin, and albumin. Being the major transport protein for thyroid hormone and that it binds $\mathrm{T} 3$ and $\mathrm{T} 4$ in a ratio of 1:1, the concentration and degree of saturation of TBG are the major determinants of fT4. Further, changes in the TBG concentration parallel that of the total T3/T4 plasma concentration; however, the same rule does not necessarily apply in the opposite direction. Thus, even though TBG may increase under specific circumstances, e.g. pregnancy or during acute hepatitis, the associated increase in the total T3 and T4 concentrations will, given time, result in a new state of equilibrium with fT4 remaining within the euthyroid window. An important exception to this rule is observed during sepsis, acute illness or after cardiopulmonary bypass surgery during which T3 and T4 are 
spliced from TBG, resulting in a significant increase in fT3 and fT4. The functional significance of this reaction is unknown, but it has been hypothesised that this process may facilitate the release of iodine for antibacterial purposes. ${ }^{11}$

TBG exhibits a 20-fold higher binding affinity for T4 compared to $\mathrm{T3}$, resulting in an approximate $\mathrm{fT} 4$ of $0.02 \%$ compared to an fT3 of $0.3 \%$. As it is only the free fractions of T3 and T4 that are biologically active, the clearance rate of thyroid hormone is determined by the metabolism of $\mathrm{T} 3$ and $\mathrm{T} 4$, and not the rate of dissociation from plasma proteins. This can be explained by the rapid influx and efflux of thyroid hormones across cell membranes. Under circumstances of increasing fT3/fT4, a state of equilibrium between the intracellular T3/T4 and the extracellular free fractions is quickly established. As such, in the absence of sufficient quantities of TBG, albeit relatively so, the intracellular fraction of T3/T4 will be maintained at a higher concentration and will elicit bolstered physiological responses, including accelerated hepatic metabolism. ${ }^{12,13}$ Therefore, as a rapid increase in fT3 and fT4 following administration of high doses of exogenous hormone results in accelerating its own clearance, it is often futile to administer supraphysiological doses of thyroxine.

\subsection{The metabolic conversion and turnover of thyroid hormone}

As alluded to earlier, the thyroid gland preferentially synthesizes T4 (in a T4:T3 ratio of approximately 15:1). From a physiological point of view, this makes sense on various levels. First, T4 demonstrates a higher binding affinity for TBG than T3. It therefore equilibrates at a lower fT4 and have a longer half-life compared to T3 (6.7 days vs $18 \mathrm{hrs}$.). It can therefore be regarded as a stable reservoir that undergoes gradual dissociation from plasma proteins as a function of changing fT3/fT4 concentrations. Second, T4 is less potent and elicits a biological response with a magnitude equal to one third of that of $\mathrm{T} 3$, reducing the risk for severe symptomology following injury or inflammation of the thyroid. Third, while T3 can be synthesized from T4 in most target tissues on a need-be basis, the reaction cannot be reversed in the case of excessive exposure to endogenous or exogenous T3. ${ }^{14,15}$

The conversion of $\mathrm{T} 4$ to $\mathrm{T} 3$, and ultimately diiodothyronine (T2), a metabolically inactive derivative, is regulated by three key enzymes, i.e. iodothyronine deiodinase types 1, 2 and $3\left(D_{1}-D_{3}\right)$. In short, T4 is converted to active T3 by $D_{1}$ and $D_{2}$, while it is inactivated by $\mathrm{D}_{3}$ via conversion to reverse T3 (rT3). $T 3$ and $r T 3$ are subsequently converted to $T 2$ by $D_{3}$ and $D_{1} / D_{2}$, respectively. In what can be regarded as one of the most striking homeostatic mechanisms in the human body, $D_{1}$ expression is upregulated, while $D_{2}$ expression is reduced, as a function of increasing T4 concentrations. ${ }^{1,14}$ It is therefore understandable that while $D_{1}$ is located in the major peripheral organs that drive the basal metabolism, i.e. liver, kidneys and thyroid gland, $D_{2}$ is expressed in organs or body systems that may be susceptible to severe pathological processes following excessive T3 exposure, i.e. the central nervous system (CNS), heart, placenta, skeletal muscle and brown adipose tissue (BAT). The opposite scenario also elicits a protective effect in that decreasing levels of $\mathrm{T} 4$, as is the case in clinical hypothyroidism, upregulate the activity of $D_{2}$, ensuring a stable supply of T3 to critical organ systems for as long as possible. Taken together, it can be anticipated that such protection will not be established following an overdose with $\mathrm{T} 3$, and that psychiatric and cardiac symptomology will swiftly present. Concerning the functional role of $D_{3}$, it is the major T3/T4 inactivating enzyme. ${ }^{16}$ As is true for $D_{1}, D_{3}$ is also upregulated by increasing concentrations of $\mathrm{T} 4$, preventing exacerbated manifestations of thyrotoxicosis to some extent. Interestingly, $D_{3}$ is expressed in high concentrations in the fetus, while postnatally it is found in the placenta, CNS, skeletal muscle and liver. Pathologically, $D_{3}$ contributes to the development of consumptive hypothyroidism, a rare condition in which the rate of T3/T4 inactivation overcomes the secretory capacity of the thyroid gland. ${ }^{17}$

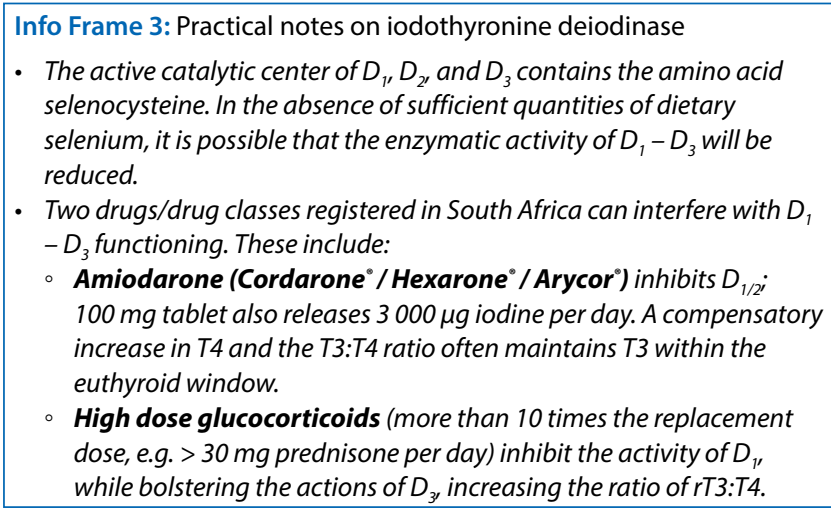

Of the approximate $800 \mu \mathrm{g}$ of extrathyroidal T4 distributed in a space of 10 liters, $10 \%(85 \mu \mathrm{g})$ is cleared daily. In contrast, as a result of its decreased affinity for TBG, T3 has a volume of distribution equal to 40 liters, of which $60 \%$ is cleared over 24 hours. Given the preferential thyroidal synthesis of T4, it is evident that the daily reduction in $\mathrm{T} 3$ concentration cannot be compensated for by the thyroid gland alone. In fact, only $15-20 \%$ of T3 originates from the thyroid gland, while $80-85 \%$ is provided by the extrathyroidal activity of $D_{1}$ and $D_{2}{ }^{18}$ Further, T4 can also be excreted via hepatic glucoronidation, a pathway induced by among others, rifampin, phenytoin and selective serotonin reuptake inhibitors. Whereas T4 secretion will adjust accordingly in patients with a functioning hypothalamuspituitary-thyroid axis, patients receiving exogenous T4 will often require an increased thyroxine dose. ${ }^{19}$

\section{Different Lighthouses, Often Different Messages: Insights into Diagnostic Measures of Altered Thyroid States}

Although much is already known about the various screening tools for thyroid hormone functioning, this section will provide some insightful perspectives on the aims, choice, and interpretation of the diagnostic tests available. Generally, a diagnosis of altered thyroid hormone status can be based on markers of aberrant hypothalamus-pituitary axis functioning, serum T3/T4 concentrations, autoimmune assault of the thyroid gland, or changes in thyroidal iodine metabolism. Rarely, assessments of the effects of thyroid hormones on target tissues 
are also used to establish the severity of thyroid hormone induced pathology.

\subsection{Measurements of TSH and serum thyroid hormone concentrations}

Whereas assessments of TSH provide an indirect, though sensitive, indication of thyroid gland functioning and the degree of its physiological impact, measurements of serum T3 and T4, notably that of fT3 and fT4, are directly related to the severity of thyroid hormone related pathology. ${ }^{1}$ For $96 \%$ of the general population, the 'normal' serum range for TSH, viz. 0.4-4.2 mU/L, is an accurate indication of an euthyroid state..$^{20,21}$ Importantly, the ten-fold difference between the lower and upper limit of the range provides some useful perspective on the large degree of between-patient variance and what the actual definition of 'normal' may entail. Although a parallel relationship between TSH and fT4 exists within the window of an euthyroid state, this merely by virtue of its action-reaction association, linear correlations under either a hypo- or hyperthyroid state can be indicative of central pathology. However, although such possibilities must be considered when a diagnosis of thyroidal pathology is deliberated, these conditions are monitored and treated under specialist supervision and will not be discussed further.

In patients without hypothalamic or pituitary dysfunction presenting with elevated levels of T4, TSH values will be decreased. Irrespective of the causal circumstance, reductions in TSH are broadly divided into two categories, i.e. values less than $0.1 \mathrm{mU} / \mathrm{L}$ and values ranging between $0.1-0.4 \mathrm{mU} / \mathrm{L}$; the former normally indicating clinical hyperthyroidism or excessive treatment with exogenous thyroid hormone. A notable exception to this rule may be observed in patients still presenting with partial thyroid function that are treated with exogenous thyroid hormone at doses equal to the normal daily physiological production, i.e. 100-150 $\mu \mathrm{g}$. This will be discussed in paragraph 3.2.

To the contrary, patients diagnosed with primary hypothyroidism may present with TSH levels ranging from marginally higher values, up to $1000 \mathrm{mU} / \mathrm{L}$. However, patients with serum TSH concentrations between 4 and $15 \mathrm{mU} / \mathrm{L}$ (often up to $50 \mathrm{mU} / \mathrm{L}$ ) normally do not suffer from clinical hypothyroidism and will often present with low-normal fT4 and normal fT 3 concentrations. This phenomenon, resulting from the compensatory mechanisms brought into play under circumstances of mild thyroid hypofunction (see Info Frame 1), is not regarded as a pathological response, but rather as a normal physiological reaction to a gradual reduction in thyroid functioning.

Quantification of serum T3/T4 is performed to substantiate the predictive value of a TSH result and both analyses are often performed simultaneously. Measurements of T3 and T4 either quantify the total or free hormone concentrations or provide an indication of the free thyroxine index (fT4I). Where the total and free concentrations of T3 and T4 are directly quantified, the $\mathrm{fT} 4 \mathrm{I}$ is a calculated value equal to the quotient of the total $\mathrm{T} 4$ and the thyroxine binding index (TBI). ${ }^{22}$ Normally, total T3/T4 concentrations are closely linked to changes in a number of anatomical and physiological parameters, e.g. body composition, reproductive status and TBG concentration. As such, and as explained in paragraph 1.2, changes in the TBG concentration are paralleled by changes in the total hormone concentration. Given that only a third of TBG binding sites are occupied under euthyroid conditions, ${ }^{23}$ a total T3/ T4 measurement is a reliable reflection of the physiological status of thyroid hormone functioning under circumstances of normal TBG concentrations. However, when changes to the TBG concentration occur, quantification of the total serum hormone pool becomes an inaccurate predictor of the clinical picture. For instance, if TBG increases, as is true during pregnancy, ${ }^{24}$ normal fT4 concentrations are maintained due to an increase in the total $\mathrm{T} 4$ and a reduction in the number of occupied TBG binding sites. In this case, measurements of total T4 will be disproportionally higher compared to the fT4 value, while the fT4I will be normal. However, should a parallel increase in fT4I and total T4 be observed, it may be indicative of over-replacement therapy or increased T4 secretion. Also, a parallel decrease is observed in patients with primary hypothyroidism where the reduced rate of T4 production will result in a reduction in the fT4, irrespective of the concentration or binding capacity of TBG. This being said, use of the fT4I has mostly been superseded by direct quantifications of fT4 as accurate detection techniques became available. Taken together, although total T3/T4 measurements may provide a picture of the complete thyroid hormone load, its physiological impact can only be interpreted with accuracy if appraised in combination with either the fT 4 or fT4I.

Although it is undeniable that the clinical heterogeneity of thyroidal pathology is considerable, and much more complicated than what can be divulged in this paper, the examples provided in Table 1 provide useful and insightful perspectives on the general diagnostic directions flagged by laboratory findings.

\subsection{Markers of autoimmune responses against the thyroid}

The two most common autoimmune syndromes targeting thyroidal physiology are Grave's disease (resulting in thyrotoxicosis) and Hashimoto's thyroiditis (HT). ${ }^{25}$ In fact, contrary to previous descriptions, these syndromes are two divergent trajectories of the same condition, i.e. chronic thyroiditis (see Section 3). ${ }^{8,26}$ Importantly, while 'thyroiditis' implies both the presence of lymphocyte infiltrate and destruction of thyroid follicles, the term 'chronic thyroiditis' was conceptualised to describe thyroidal lymphocyte infiltration that neither includes, nor excludes a role for follicle destruction. Chronic thyroiditis is marked by the presence of autoimmune antibodies targeted against three fundamental constructs of thyroid hormone synthesis, i.e. TSHR (TSHR-Ab), TPO (TPO-Ab) and Tg (Tg-Ab).

Interestingly, while TSHR-Abs are exclusively found in patients with active autoimmune thyroiditis (or a recent history of such illness), TPO-Abs and Tg-Abs are commonly quantified in the serum of healthy individuals, five times more so in women than in men. ${ }^{27}$ Further, TSHR-Abs can broadly be divided in agonising, antagonising or neutral clusters. While the antagonising and neutral clusters are demonstrated in $10-20 \%$ of patients with 
Table 1. Practical examples of expected TSH and T3/T4 levels determined by laboratory assessment

\begin{tabular}{|c|c|c|c|c|}
\hline Scenario & TSH & Total T3/T4 & fT3/T4/ fT4I & Notes \\
\hline $\begin{array}{l}\text { Primary hypothyroidism, i.e. iodine } \\
\text { deficiency, Li+ use, Hashimoto's disease }\end{array}$ & $\uparrow$ & $\downarrow$ & $\leftrightarrow(\downarrow)$ & $\begin{array}{l}\text { Compensatory mechanisms will adjust for a gradual } \\
\text { decrease in total T3/T4 (see Info Frame 1), ultimately } \\
\text { failing if clinical prognosis deteriorates. }\end{array}$ \\
\hline $\begin{array}{l}\text { Primary hyperthyroidism, i.e. Grave's } \\
\text { disease, inflammatory thyroid, Plummer's } \\
\text { disease }\end{array}$ & $\downarrow$ & $\uparrow$ & $\uparrow$ & $\begin{array}{l}\text { Delayed adjustment of TBG concentrations result in } \\
\text { saturation of available binding sites and subsequent } \\
\text { increase in fT3/T4. }\end{array}$ \\
\hline Over-replacement therapy & $\downarrow$ & $\uparrow$ & $\uparrow$ & See 'primary hyperthyroidism' above \\
\hline $\begin{array}{l}\text { Replacement analogous to the normal } \\
\text { daily synthesis rate of } \mathrm{T} 4 \text {, as opposed } \\
\text { to adjusted therapy, in patients with a } \\
\text { partially functional thyroid, i.e. sub-clinical } \\
\text { hypothyroidism }\end{array}$ & $\downarrow$ & $\leftrightarrow$ & $\leftrightarrow$ & $\begin{array}{l}\text { In patients with sub-clinical hypothyroidism who are } \\
\text { treated with a thyroxine dose equal to the normal daily } \\
\text { production, i.e. } 100-200 \mu \mathrm{g} \text { per day, mildly elevated } \\
\text { pre-treatment TSH values }(0.1-0.4 \mathrm{mU} / \mathrm{L}) \text { will be } \\
\text { reduced to levels near to the lower limit of detection. }\end{array}$ \\
\hline $\begin{array}{l}\text { Physiological state during pregnancy, } \\
\text { supraphysiological estrogen status } \\
\text { (i.e. obesity, excessive use of estrogen } \\
\text { replacement therapy), HIV-infection }\end{array}$ & $\leftrightarrow$ & $\uparrow$ & $\leftrightarrow$ & $\begin{array}{l}\text { Increased total T3/T4 is an inaccurate predictor of the } \\
\text { physiological state. An increased production of TBG is } \\
\text { the driving factor underlying an increase in total T3/ } \\
\text { T4. This can be regarded as a compensatory response, } \\
\text { resulting in the maintenance of an euthyroid state. }\end{array}$ \\
\hline $\begin{array}{l}\text { Acute illness, use of large doses of } \\
\text { glucocorticoids, nephrotic syndrome, } \\
\text { among others }\end{array}$ & $\downarrow$ & $\leftrightarrow(\downarrow)$ & $\uparrow$ & $\begin{array}{l}\text { Driving factor is rapid dissociation between TBG and } \\
\text { T3/T4. Depending on the duration of such triggering } \\
\text { factor, metabolism of the free fraction and the rate } \\
\text { of response of the hypothalamus-pituitary axis, a } \\
\text { reduction in the total serum hormone concentration } \\
\text { can be observed over time. }\end{array}$ \\
\hline
\end{tabular}

Abbreviations: TSH: thyrotropin; T3: triiodothyronine; T4: thyroxine; fT4/T3: free hormone concentration; fT4l: free thyroxine index; TBG: thyroid binding globulin; arrows in superscript brackets indicate possible outcomes following chronic disease states.

$\mathrm{HT}$, the agonising cluster is found in $80-95 \%$ of patients with Grave's disease. However, TPO-Abs and Tg-Abs are expressed in $80-100 \%$ of patients with goitrous or atrophic HT, while only being present in $50-70 \%$ of Grave's patients. ${ }^{1}$ Importantly, while TSHR-Abs play an important etiological role in both syndromes, TPO-Abs and Tg-Abs are believed to be secondary consequences of established thyroidal injury. However, they may contribute to the duration of illness. As opposed to the monoclonal nature of the TSHR-Ab, ${ }^{28}$ the polyclonality of TPO-Abs and Tg-Abs prevents it from contributing to disease pathogenesis and transferring illness from mother to offspring. Therefore, while the presence of TPO-Abs and Tg-Abs in patients without thyroiditis remains a risk factor for disease in families with a history of thyroiditis, their presence is not necessarily reason for clinical concern. ${ }^{29}$

\section{An Altered State of Equilibrium: Clinical Perspectives on Thyroid Hormone Dysregulation}

\subsection{A concise epidemiological and etiopathological comparison of two contrasting conditions}

\section{Epidemiology and conceptual description}

Although Section 3 will focus on an understanding of and approach to hypothyroidism, the pathology of hypo- and hyperthyroidism often transpire on a single continuum which will be summarised here. Both terms are broad umbrella labels used to describe decreased and increased physiological thyroid hormone concentrations, respectively. However, they indicate neither etiology nor pathogenic course. Although statistics differ depending on study locale, overt (clinical) hypothyroidism is diagnosed in $0.3 \%$ of women and $0.06 \%$ of men while hyperthyroidism is described in $0.04 \%$ of women and $0.01 \%$ of men. ${ }^{30}$ However, subclinical, i.e. asymptomatic, hypothyroidism is prevalent in $3-21 \%$ of the general population, ${ }^{27}$ while subclinical hyperthyroidism is observed in $\sim 3 \%$ of individuals. ${ }^{30}$ Importantly, studies investigating the prevalence of subclinical hyperthyroidism often do not exclude cases of exogenous thyroxine use, arguably contributing to the incidence rates reported. ${ }^{30}$ Given that no such caveat exists in the case of hypothyroidism, data pertaining to sub-clinical hypothyroidism may be a more accurate reflection of the current scenario. Further, whereas the most common causes of hyperthyroidism are related to autoimmune responses that often require specialist supervision, hypothyroidism may also occur spontaneously over time and can be triggered by a number of factors other than autoimmune assault, e.g. iodine deficiency, old age, or drug use.

\section{Pathological and symptomological summary}

As a result of sensitive and regularly routine TSH assessment and that inexpensive thyroxine preparations are readily available, overt hypothyroidism is not often described in clinical practice anymore. Although clinical hyperthyroidism of at least modest severity is observed in patients with Grave's disease, rapid diagnosis and treatment also contributes to dampening the symptomology seen in modern medicine. Table 2 provides a concise overview of the major symptomological constructs of each condition.

Except for the symptoms that transpire following rapid withdrawal of thyroxine therapy, the onset of hypothyroid symptoms is often so slow that it may only become evident after years of progressive thyroid failure. As alluded to earlier, overt hypothyroidism is rarely described today, while sub-clinical hypothyroidism, as evinced by slight elevations in serum TSH concentrations, and not hypothyroid symptomology, is diagnosed in $3-21 \%$ of patients, depending on study locale, gender and age. 27,30 


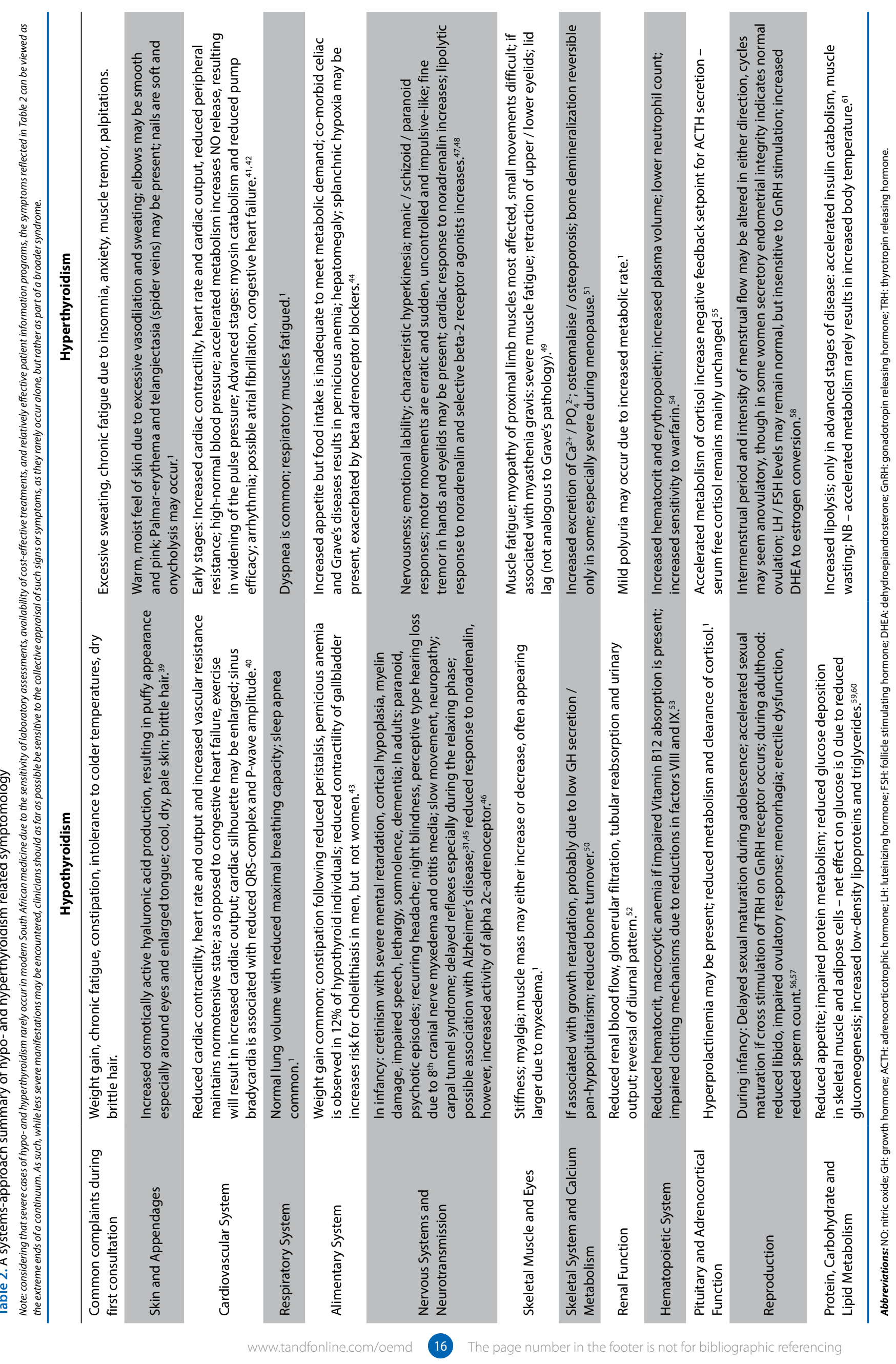


In areas of the world where the dietary intake of iodine is sufficient, the most common cause of hypothyroidism is $\mathrm{HT}^{26}$ Being the thyroid-inhibiting trajectory of chronic thyroiditis, ${ }^{8} \mathrm{HT}$ is characterised by the presence of atrophic TSHR antagonising antibodies. However, given the molecular overlap between HT and hyperthyroidic Grave's disease, it is not surprising that some patients with HT can develop Graves's orbitopathy, while patients with Grave's disease can progress to clinical hypothyroidism. Further, risk factors for HT and Grave's disease overlap and include pregnancy, the use of iodine and iodine containing medicine, cytokine treatment, radiation therapy and systemic infection. ${ }^{26}$ While a comprehensive review of the pathophysiology of these conditions falls outside the scope of the current paper, it suffices to say that autoimmune assault of the thyroid gland in either direction, will elicit clinical symptomology in the absence of sufficient treatment. Indeed, as opposed to iatrogenic hypothyroidism or transient inflammatory responses, autoimmune disease is often progressive. ' Other rare causes of clinical hypothyroidism that will necessitate treatment include congenital thyroidal defects, ${ }^{31}$ dysfunctional conversion of T4 to $\mathrm{T}^{32}$ (not prevalent in South Africa), central hypothyroidism, ${ }^{33}$ and receptor resistance to thyroid hormone. ${ }^{34}$

This being said, the incidence of sub-clinical hypothyroidism significantly exceeds that of its clinical counterpart. ${ }^{30}$ Asymptomatic hypothyroidism transpires spontaneously ${ }^{1}$ or results from either iodine deficiency or excess (refer to Info Frame 1), 35,36 the use of thyroid compromising pharmacotherapy, e.g. lithium and ethionamide, ${ }^{37}$ dietary intake of goitrogens, e.g. thiocyanate containing cabbage, ${ }^{1}$ and cigarette smoking, especially in patients with underlying autoimmune thyroid disease. ${ }^{38}$ However, such cases of hypothyroidism are mostly reversible following corrective action.

\subsection{Therapeutic approaches to hypothyroidism}

In cases where corrective action can be taken to reverse the hypothyroid prognosis, e.g. withdrawal or cessation of iatrogenic triggers, supplementing iodine according to the recommended daily intake and adjusting dietary consumption of goitrous ingredients, such approaches will be the first choice of intervention. However, most circumstances necessitate the use of exogenous thyroxine, which will be discussed here.

\section{Physiology and pharmacology of exogenous thyroxine}

Exogenous thyroxine is a pivotal construct in the treatment of both clinical and sub-clinical hypothyroidism. With a biological half-life of 7 days under an euthyroid state and a bioavailability of $80 \%$ following oral dosing, ${ }^{62}$ thyroxine remains effective even when doses are occasionally skipped. Further, an euthyroid state can also be induced with higher weekly, as opposed to lower daily dosages. A reliable and validated guideline for calculating the appropriate thyroxine dose in clinical hypothyroidism is $1.6-1.8 \mu \mathrm{g} / \mathrm{kg} /$ day estimated with respect to the ideal target lean body mass, with increasing concentrations in obesity not being advocated. ${ }^{63}$ Due to thyroxine being $80 \%$ bioavailable, the calculated dose will normally be near $20 \%$ more than the theoretical endogenous synthesis rate. Further, slight deviations from the exact dose required by physiological demand in either direction are almost always inconsequential as a number of compensatory mechanisms are brought into play (see Info Frame 1 and Sections 1 and 2). Importantly, although the bulk of oral thyroxine is absorbed from the stomach and small intestine, gastric acid secretion is a necessary requirement. Indeed, patients being treated with proton pump inhibitors, e.g. esomeprazole may require upward dose adjustments of up to $22-34 \% .{ }^{64}$

Considering that $\mathrm{T} 3$ is regarded as the metabolically active moiety of T4, a number of factors must be contemplated with respect to exogenous T3 treatment and the interplay between T3 and T4. First, under physiological conditions, $80 \%$ of T3 is converted from $T 4$ by $D_{1}$ and $D_{2}$, while the thyroid itself is responsible for secreting the remaining $20 \% .^{65}$ Further, following an oral dose of $100 \mu \mathrm{g}$ thyroxine, $80 \%(\sim 80 \mu \mathrm{g})$ is absorbed of which $\sim 36 \%(\sim 30 \mu \mathrm{g})$ is converted to T3. As such, the ratio of T3:T4 in patients exclusively dependent on exogenous T4 is about $20 \%$ lower, compared to that of non-clinical individuals. This often results in a slightly higher T4 dose needed to normalise the TSH concentration, consequentially increasing the fT4 level (still, see below). ${ }^{66}$ However, where no clinical caveat exists that would suggest a different approach to be taken (see Sections 1 and 3 , and below), gradual upward titration of thyroxine doses to normalise TSH levels is standard practice, while no clinical evidence has yet been presented to demonstrate that combined T3/T4 therapy attempting to circumvent this approach is successful or more advantageous. ${ }^{67}$ In fact, T4 replacement based on body weight and fT4 levels has been demonstrated superior to combined T3/T4 therapy with respect to improvements in most markers of thyroid function. ${ }^{68}$ Further, if the biological potency and short half-life of T3 is taken into account and seeing that no protection can be exercised by $D_{2}$ against its effects in vital organ systems such as the brain, cardiac muscle and placenta, no clinical or practical reason exists for the use of $\mathrm{T} 3$ in the majority of patients consulted in primary care facilities. In fact, T3 therapy must be reserved for severe cases of thyroid related pathology diagnosed and treated under specialist supervision, whether being applied as an inhibitor or potentiator of thyroid function.

\section{Initiation of thyroxine treatment}

Initiation of thyroxine therapy depends on patient age, underlying pathologies, symptom severity, and the general health of the individual. ${ }^{1}$ In patients with mild to moderate serum TSH elevations of 5-50 mU/L (note the large degree of variance), an initial dose of $1.7 \mu \mathrm{g} / \mathrm{kg} /$ day can be administered. Both patient and clinician must be sensitive to the fact that the total and free serum T3/T4 concentrations will increase gradually, while it can take up to 6-12 weeks before normalised T3/T4 and TSH values are observed. As such, it is highly unlikely that adverse effects will be experienced during the initial phases of hormone replacement. In older patients, and those with established or a history of cardiac pathology, the initial dose must be reduced to $0.8 \mu \mathrm{g} / \mathrm{kg} /$ day and titrated upwards every $2-3$ months with $12.5 \mu \mathrm{g}$. The initial phase of drug therapy is regarded complete once TSH levels have reduced and fT4 levels increased to normal or high-normal levels. The first evaluation of such response must 
only be performed after the first 6 weeks of treatment. ${ }^{69}$ In the rare instance where children between 8 and 12 years of age have to be initiated on treatment, pseudotumor cerebri may develop in severely hypothyroid individuals started on even mild doses of thyroxine. In this case, downward titration of the thyroxine dose is not necessary as the condition responds to treatment with acetazolamide and dexamethasone. ${ }^{70}$ If a rapid reversal of hypothyroid symptoms is necessitated, i.e. myxedema coma, $25 \mu \mathrm{g}$ T4 can be administered orally every 12 hours for 24-36 hours, followed by a daily oral dose not exceeding $1.7 \mu \mathrm{g} / \mathrm{kg} /$ day. In such severe circumstances, a sudden bolstering in metabolic activity can overcome the compensatory effects of a suppressed adrenocortical reserve. In this case, hydrocortisone can be administered at a rate of $5 \mathrm{mg} /$ hour over the first 12-24 hours. ${ }^{1}$

\section{Aspects related to the monitoring of thyroxine therapy}

As explained above, measurements of TSH and / or fT4 may be used to monitor and adjust thyroxine therapy. ${ }^{1}$ However, a few caveats must be considered when evaluating laboratory results. First, in patients with central hypothyroidism, TSH levels are for apparent reasons not an accurate marker of appropriate dosing. In this case, fT4 levels within the normal to upper normal range must be the therapeutic target. Second, although TSH levels are an accurate predictor of fT4, exceptions to the rule do exist (some encountered more often than not). For example, in patients with a partially functioning thyroid gland presenting with sub-clinical hypothyroidism as evinced by serum TSH concentrations between 5 and $15 \mathrm{mU} / \mathrm{L}$ (and up to $50 \mathrm{mU} / \mathrm{L}$ in some cases), clinicians may opt to treat patients with replacement doses equal to the normal daily T4 production, viz. $1.6-1.8 \mu \mathrm{g} / \mathrm{kg} / \mathrm{day}$. This approach is often more convenient as most of the triggering factors for gradual thyroidal decline, i.e. thyroid compromising pharmacotherapy, autoimmune assault, and iodine deficiency, alter thyroid function to a varying extent over time. As such, it may be problematic to gradually titrate the dose over time, as the endogenous capability of the thyroid gland may change over time. In this case, TSH levels will often be lower than $0.01 \mathrm{mU} / \mathrm{L}$. However, while the endogenous thyroid function is suppressed due to the low levels of TSH, both total and free T3/T4 will be within the normal range (see Table 1). This approach can be regarded as safe and effective. A third example where measurements of TSH may be a counterproductive means of establishing therapeutic efficacy is found in sub-clinical hypothyroid patients presenting with anti-thyroid antibodies and serum TSH concentrations exceeding $10 \mathrm{mU} / \mathrm{L}$. Indeed, it has been shown that serum TSH concentrations in these patients may remain higher than normal, even though the total and free T3/T4 concentrations have been normalized. ${ }^{40}$ In such instances, further increases in the T4 dose with the sole purpose of lowering TSH concentrations are not advised.

Every so often, individuals are encountered claiming to need more than the normal T4 requirement of $1.6-1.8 \mu \mathrm{g} / \mathrm{kg} / \mathrm{day}$. In fact, clinicians and pharmacists will be aware of patients of average weight using up to $400 \mu \mathrm{g}$ thyroxine per day. In such cases, it will be paramount to implement downward titration of the dose to the recommended physiological requirement. Normally, this practice is implemented due to poor patient adherence during the initial stages of treatment and that is unknown to the clinician. It can also be related to attempts to decrease TSH concentrations to normal levels, even when circumstantial factors indicate this to be inappropriate. It is possible that laboratory assessments of thyroid hormone status in these patients may closely resemble those of patients taking bio-equivalent replacement doses, indeed motivating both clinician and patient to continue with excessive replacement. However, taken from Section 1, such results are founded on thyroid hormone accelerating its own metabolism and excretion over time. As an increase in fT4 during the early stages of supraphysiological 'replacement' therapy will quickly establish a new equilibrium between the intra- and extracellular free concentrations, the hepatic metabolic activity will be bolstered. As the daily intake of exogenous thyroxine will remain the same, e.g. 400 ug, a new homeostatic equilibrium will be maintained, but at a higher metabolic level. Therefore, although the fT4 may not necessarily be profoundly high, it is fundamental that both clinician and patient be aware that such doses of thyroxine establish a new physiological equilibrium at such a metabolic level that it is associated with potentially severe side-effects (summarised under 'hyperthyroidism' in Table 2), especially during chronic 'replacement therapy'.

\section{Conclusion}

The current paper briefly summarised important, but often overlooked aspects of thyroid hormone physiology, iodine metabolism and hypothyroidism. While a broad focus has been placed on different aspects of these constructs that are of interest to clinicians consulting in primary practice, the following key highlights can be taken from this review:

- The recommended daily requirement of iodine varies between 90 and $200 \mu \mathrm{g} /$ day. Use of concentrations higher than this is associated with thyroid dysregulation and are strongly discouraged, except if employed for thyrotoxicosis (see Info Frame 1).

- While the human body can compensate for mild to moderate reductions in iodine, the same is not true with respect to the opposite scenario.

- Although TSH is an appropriate diagnostic parameter to establish thyroid status and therapeutic efficacy, several factors must be kept in mind when evaluating a TSH result. For instance, TSH may be reduced during acute illness or during replacement therapy with thyroxine doses equal to the normal daily production rate in patients with a partial functioning thyroid. Neither circumstance necessitates corrective action. Also, in some patients, the negative feedback set-point for TSH secretion may be established at a disproportionally higher total and free T4 concentration than the normal values, in which case further upward titration of T4 is not advised.

- During acute illness, sepsis or trauma, an increase in the fT3/ T4 may be observed. This is normal and does not require corrective steps to be taken.

- The majority of hypothyroid cases can be described as subclinical. Such diagnoses are based on slight to moderate 
elevations in TSH that can range between 5 and $50 \mathrm{mU} / \mathrm{L}$ and low-normal to normal total and free T3/T4, without any clear sign or symptom of hypothyroid pathology. It is debatable whether such patients need treatment, although thyroxine supplementation or replacement in such scenarios are not harmful.

- Hashimoto's thyroiditis and Grave's disease can be regarded as two opposing ends of a single continuum. In families where markers of autoimmune disease of the thyroid are demonstrated, it is possible that assault of the thyroid gland can transpire in either direction.

- No clinical evidence exists that advocates a therapeutic role for $\mathrm{T} 3$ in the majority of patients seen in primary practice. In fact, replacement with $\mathrm{T} 4$ based on body weight and $\mathrm{fT} 4$, is superior to treatment with T3 with respect to all markers of thyroid function. T3 should be reserved for specialist use under severe, but regulated circumstances, i.e. patients admitted in hospital.

- Althoughsomepatients believethey requiresupraphysiological doses of thyroxine, i.e. $>200 \mu \mathrm{g} /$ day, no clinical evidence exists to suggest this to be appropriate. Where high-normal fT4 concentrations are demonstrated in these instances, it can almost always be ascribed to a higher metabolic clearance rate. Such practices are harmful and patients should be referred for specialist supervision.

\section{References}

1. Salvatore D, Davies T, M S, et al. Thyroid. In: Melmed S, Polonsky K, Larsen P, Kronenberg H, eds. Williams Textbook of Endocrinology. 12 ed. Philadelphia: Elsevier Saunders; 2011: 1897.

2. Emerson $\mathrm{CH}$, Anderson AJ, Howard WJ, Utiger RD. Serum thyroxine and triiodothyronine concentrations during iodide treatment of hyperthyroidism. J Clin Endocrinol Metab. 1975;40(1):33-6.

3. Wolff J, Chaikoff I. Plasma inorganic iodide as a homeostatic regulator of thyroid function. J Biol Chem. 1948;174:555-64.

4. Eng PH, Cardona GR, Fang S-L, et al. Escape from the acute Wolff-Chaikoff effect is associated with a decrease in thyroid sodium/iodide symporter messenger ribonucleic acid and protein. Endocrinology. 1999;140(8):3404-10.

5. Abuid J, Larsen P. Triiodothyronine and thyroxine in hyperthyroidism comparison of the acute changes during therapy with antithyroid agents. J Clin Invest. 1974;54(1):201.

6. Larsen PR. Thyroidal triiodothyronine and thyroxine in Graves' disease: correlation with presurgical treatment, thyroid status, and iodine content. J Clin Endocrinol Metab. 1975;41(6):1098-1104.

7. Bizhanova A, Kopp P. The sodium-iodide symporter NIS and pendrin in iodide homeostasis of the thyroid. Endocrinology. 2009;150(3):1084-90.

8. Davies TF, Amino N. A new classification for human autoimmune thyroid disease. Thyroid. 1993;3(4):331-3.

9. Brabant G, Prank K, Ranft U, et al. Circadian and pulsatile TSH secretion under physiological and pathophysiological conditions. Horm Metab Res. 1990;23(SUPPL):12-17.

10. Brabant G, Prank K, Ranft U, et al. Physiological regulation of circadian and pulsatile thyrotropin secretion in normal man and woman. J Clin Endocrinol Metab. 1990;70(2):403-9.

11. Pemberton $P$, Stein $P$, Pepys $M$, Potter J, Carrell R. Hormone binding globulins undergo serpin conformational change in inflammation. Nature. 1988;336(6196):257-8.

12. Robbins J, Rall JE. The interaction of thyroid hormones and protein in biological fluids. Recent Prog Horm Res. 1957;13:161.

13. Mendel CM. The free hormone hypothesis: a physiologically based mathematical model. Endocr Rev. 1989;10(3):232-74.

14. Bianco AC, Salvatore D, Gereben B, Berry MJ, Larsen PR. Biochemistry, cellular and molecular biology, and physiological roles of the iodothyronine selenodeiodinases. Endocr Rev. 2002;23(1):38-89.
15. Galton VA, de Waard E, Parlow AF, St Germain DL, Hernandez A. Life without the iodothyronine deiodinases. Endocrinology. 2014;155(10):4081-7.

16. Huang SA. Physiology and pathophysiology of type 3 deiodinase in humans. Thyroid. 2005;15(8):875-81

17. Huang SA, Tu HM, Harney JW, et al. Severe hypothyroidism caused by type 3 iodothyronine deiodinase in infantile hemangiomas. N Engl J Med. 2000;343(3):185-9.

18. Saberi $M$, Sterling $F$, Utiger R. Reduction in extrathyroidal triiodothyronine production by propylthiouracil in man. J Clin Invest. 1975;55(2):218.

19. Wu S-y, Green WL, Huang W-s, Hays MT, Chopra IJ. Alternate pathways of thyroid hormone metabolism. Thyroid. 2005;15(8):943-58.

20. Surks MI, Goswami G, Daniels GH. The thyrotropin reference range should remain unchanged. J Clin Endocrinol Metab. 2005;90(9):5489-96.

21. Wartofsky L, Dickey RA. The evidence for a narrower thyrotropin reference range is compelling. J Clin Endocrinol Metab. 2005;90(9):5483-8.

22. Faix JD, Rosen HN, Velazquez FR. Indirect estimation of thyroid hormone-binding proteins to calculate free thyroxine index: comparison of nonisotopic methods that use labeled thyroxine ("T-uptake"). Clin Chem. 1995;41(1):41-7.

23. Sapin $R$, d'Herbomez $M$. Free thyroxine measured by equilibrium dialysis and nine immunoassays in sera with various serum thyroxine-binding capacities. Clin Chem. 2003;49(9):1531-5.

24. Glinoer D. The regulation of thyroid function in pregnancy: pathways of endocrine adaptation from physiology to pathology. Endocr Rev. 1997;18(3):404-33.

25. Rapoport B, McLachlan SM. Thyroid autoimmunity. J Clin Invest 2001;108(9):1253

26. Pearce EN, Farwell AP, Braverman LE. Thyroiditis. N Engl J Med 2003;348(26):2646-55

27. Hollowell JG, Staehling NW, Flanders WD, et al. Serum TSH, T4, and thyroid antibodies in the United States population (1988 to 1994): National Health and Nutrition Examination Survey (NHANES III). J Clin Endocrinol Metab. 2002;87(2):489-99.

28. Valente WA, Vitti $P$, Yavin $Z$, et al. Monoclonal antibodies to the thyrotropin receptor: stimulating and blocking antibodies derived from the lymphocytes of patients with Graves disease. Proceedings of the National Academy of Sciences. 1982;79(21):6680-4.

29. Vanderpump M, Tunbrldge W, French J, et al. The incidence of thyroid disorders in the community: a twenty-year follow-up of the Whickham Survey. Clin Endocrinol. 1995;43(1):55-68.

30. Vanderpump MP, Tunbridge W. The epidemiology of thyroid diseases. Werner and Ingbar's the thyroid: a fundamental and clinical text. 2005:398-406.

31. Kratzsch J, Pulzer F. Thyroid gland development and defects. Best Pract Res Clin Endocrinol Metab. 2008;22(1):57-75.

32. Canani LH, Capp C, Dora JM, et al. The type 2 deiodinase A/G (Thr92Ala) polymorphism is associated with decreased enzyme velocity and increased insulin resistance in patients with type 2 diabetes mellitus. J Clin Endocrinol Metab. 2005;90(6):3472-8.

33. Comi R, Gesundheit N, Murray L, Gorden P, Weintraub B. Response of thyrotropin-secreting pituitary adenomas to a long-acting somatostatin analogue. N Engl J Med. 1987;317(1):12-17.

34. Refetoff S, Dumitrescu AM. Syndromes of reduced sensitivity to thyroid hormone: genetic defects in hormone receptors, cell transporters and deiodination. Best Pract Res Clin Endocrinol Metab. 2007;21(2):277-305.

35. Zimmermann MB. lodine deficiency. Endocr Rev. 2009;30(4):376-408.

36. Martino E, Bartalena L, Bogazzi F, Braverman LE. The effects of amiodarone on the thyroid. Endocr Rev. 2001;22(2):240-54.

37. Stagnaro-Green A. Postpartum thyroiditis. Best Pract Res Clin Endocrinol Metab. 2004;18(2):303-16.

38. Müller B, Zulewski H, Huber P, Ratcliffe JG, Staub J-J. Impaired action of thyroid hormone associated with smoking in women with hypothyroidism. N Engl J Med. 1995;333(15):964-9.

39. Smith TJ, Bahn RS, Gorman CA. Connective Tissue, Glycosaminoglycans, and Diseases the Thyroid. Endocr Rev. 1989;10(3):366-91.

40. Biondi B, Cooper DS. The clinical significance of subclinical thyroid dysfunction. Endocr Rev. 2007;29(1):76-131.

41. Napoli R, Biondi B, Guardasole V, et al. Impact of hyperthyroidism and its correction on vascular reactivity in humans. Circulation. 2001;104(25):3076-80. 
42. Fazio S, Palmieri EA, Lombardi G, Biondi B. Effects of thyroid hormone on the cardiovascular system. Recent Prog Horm Res. 2004;59(1):31-50.

43. Völzke $H$, Robinson DM, John U. Association between thyroid function and gallstone disease. World Journal of Gastroenterology: WJG. 2005;11(35):5530.

44. Myers J, Brannon E, Holland B. A correlative study of the cardiac output and the hepatic circulation in hyperthyroidism. J Clin Invest. 1950;29(8):1069.

45. Williams G. Neurodevelopmental and neurophysiological actions of thyroid hormone. J Neuroendocrinol. 2008;20(6):784-794.

46. Haluzik M, Nedvidkova J, Bartak V, et al. Effects of hypo-and hyperthyroidism on noradrenergic activity and glycerol concentrations in human subcutaneous abdominal adipose tissue assessed with microdialysis. J Clin Endocrinol Metab. 2003;88(12):5605-8.

47. Coulombe P, Dussault J, Walker P. Plasma catecholamine concentrations in hyperthyroidism and hypothyroidism. Metabolism. 1976;25(9):973-9.

48. Hellström L, Wahrenberg H, Reynisdottir S, Arner P. Catecholamine-induced adipocyte lipolysis in human hyperthyroidism. J Clin Endocrinol Metab. 1997;82(1):159-66.

49. Marinò $M$, Barbesino $G$, Pinchera $A$, et al. Increased frequency of euthyroid ophthalmopathy in patients with Graves' disease associated with myasthenia gravis. Thyroid. 2000;10(9):799-802.

50. Murphy E, Williams GR. The thyroid and the skeleton. Clin Endocrinol. 2004;61(3):285-98.

51. Faber J, Galløe AM. Changes in bone mass during prolonged subclinical hyperthyroidism due to L-thyroxine treatment: a meta-analysis. Eur J Endocrinol. 1994;130(4):350-6.

52. Iwasaki Y, Oiso $Y$, Yamauchi K, et al. Osmoregulation of plasma vasopressin in myxedema. J Clin Endocrinol Metab. 1990;70(2):534-9.

53. Squizzato A, Romualdi E, Buller H, Gerdes V. Thyroid dysfunction and effects on coagulation and fibrinolysis: a systematic review. J Clin Endocrinol Metab. 2007;92(7):2415-20.

54. Erem C, Ersoz H, Karti S, et al. Blood coagulation and fibrinolysis in patients with hyperthyroidism. J Endocrinol Invest. 2002;25(4):345-50.

55. Taniyama M, Honma K, Ban Y. Urinary cortisol metabolites in the assessment of peripheral thyroid hormone action: application for diagnosis of resistance to thyroid hormone. Thyroid. 1993;3(3):229-33.

56. Yoshimura M, Hershman JM. Thyrotropic action of human chorionic gonadotropin. Thyroid. 1995;5(5):425-34.
57. Redmond GP. Thyroid dysfunction and women's reproductive health. Thyroid. 2004;14(3), Suppl1:5-15.

58. Meikle AW. The interrelationships between thyroid dysfunction and hypogonadism in men and boys. Thyroid. 2004;14(3) Suppl 1:17-25.

59. Mullur R, Liu Y-Y, Brent GA. Thyroid hormone regulation of metabolism. Physiol Rev. 2014;94(2):355-82.

60. Crunkhorn S, Patti M-E. Links between thyroid hormone action, oxidative metabolism, and diabetes risk? Thyroid. 2008;18(2):227-37.

61. Silva JE. The thermogenic effect of thyroid hormone and its clinical implications. Ann Intern Med. 2003;139(3):205-13.

62. Toft AD. Thyroxine therapy. N Engl J Med. 1994;331(3):174-80.

63. Santini F, Pinchera A, Marsili A, et al. Lean body mass is a major determinant of levothyroxine dosage in the treatment of thyroid diseases. J Clin Endocrinol Metab. 2005;90(1):124-7.

64. Centanni M, Gargano L, Canettieri G, et al. Thyroxine in goiter, Helicobacter pylori infection, and chronic gastritis. N Engl J Med. 2006;354(17):1787-95.

65. Escobar-Morreale HF, Obregón MJ, Del Rey FE, De Escobar GM. Replacement therapy for hypothyroidism with thyroxine alone does not ensure euthyroidism in all tissues, as studied in thyroidectomized rats. J Clin Invest. 1995;96(6):2828.

66. Jonklaas J, Davidson B, Bhagat S, Soldin SJ. Triiodothyronine levels in athyreotic individuals during levothyroxine therapy. JAMA. 2008;299(7):769-77.

67. Grozinsky-Glasberg S, Fraser A, Nahshoni E, Weizman A, Leibovici L. Thyroxinetriiodothyronine combination therapy versus thyroxine monotherapy for clinical hypothyroidism: meta-analysis of randomized controlled trials. J Clin Endocrinol Metab. 2006;91(7):2592-9.

68. Slawik M, Klawitter $\mathrm{Br}$, Meiser $\mathrm{E}$, et al. Thyroid hormone replacement for central hypothyroidism: a randomized controlled trial comparing two doses of thyroxine (T4) with a combination of T4 and triiodothyronine. J Clin Endocrinol Metab. 2007;92(11):4115-22.

69. Carr D, McLeod D, Parry G, Thornes $H$. Fine adjustment of thyroxine replacement dosage: comparison of the thyrotrophin releasing hormone test using a sensitive thyrotrophin assay with measurement of free thyroid hormones and clinical assessment. Clin Endocrinol. 1988;28(3):325-33.

70. Van Dop C, Conte FA, Koch TK, Clark SJ, Wilson-Davis SL, Grumbach MM. Pseudotumor cerebri associated with initiation of levothyroxine therapy for juvenile hypothyroidism. N Engl J Med. 1983;308(18):1076-80. 